\title{
P105 Photoplethysmographic Signals Analysis Before, During, and After the Finnish Sauna Exposures
}

\author{
Matti Huotari* \\ University of Oulu, Oulu, Finland
}

\begin{abstract}
Wellness and healthiness depend on physiology if no illness exists in the body. As a treatment either in healthy or sick state, we suggest that sauna exposure could be good for cardiovascular disease (CVD) prevention, especially heart and brains arteries. We measured by our photoplethysmographic (PPG) device the responses caused by sauna exposure on the healthy males and females $(10+7)$. As a working hypothesis, the sauna exposure could lower blood pressure and refresh the arterial wall facilitating the blood flow. The mechanisms as a whole have not been enough accurately documented. We recorded for $30 \mathrm{~min}$ in the $80^{\circ} \mathrm{C}$ dry sauna conditions the PPG signals for calculation of arterial elasticity (AE), and heart rate (HR) based on inter beat interval (IBI, the first-order difference), inter beat interval variability (IBIV, the second order difference), and heart rate variability (HRV). IBI and IBIV signals were determined on the second order derivatives of photoplethysmographic (SDPPG) waveforms. For the both IBI and IBIV signals, we plotted them as Poincare plots (Figure 1) by OriginPro2019b (OriginLab). Based on the SDPPG signals from the finger and toe maxima $\mathrm{A}$ and $\mathrm{B}$ (the first maximum and minimum) we calculated both the EA for finger and toe arteries. The PPG waveforms were noiseless because the subjects were at rest and no extra morphologies exists in PPGs during the sauna exposures, like before and after sauna. In our knowledge, this is the first PPGs measured during the actual sauna exposures.
\end{abstract}

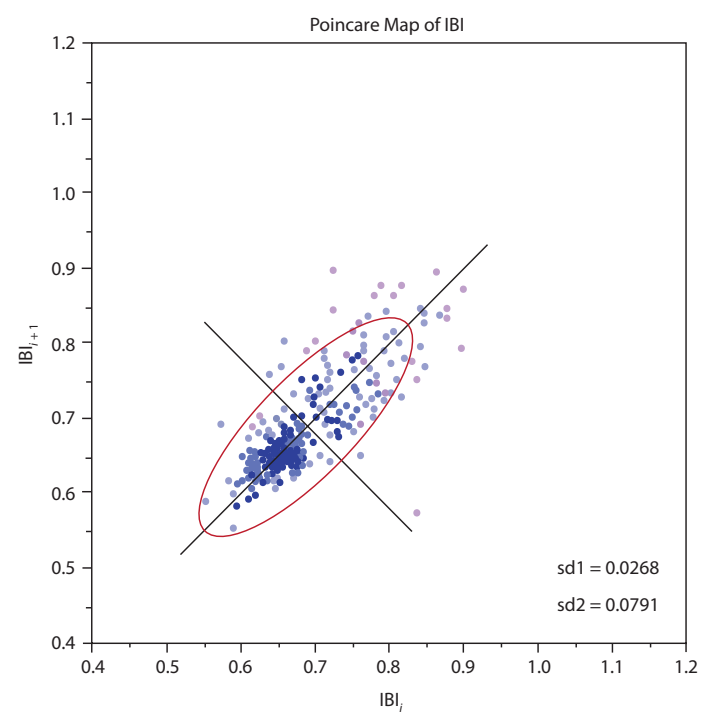

Figure $1 \mid$ Poincare plot after the sauna exposure.

\section{REFERENCES}

[1] Ischaemic heart disease still the cause of one in five deaths for men. Helsinki: Statistics Finland [referred: 23.6.2019].

[2] Kamioka H, Mori Y, Nagata K, Iwanaga S, Uzura M, Yamaguchi S. Relationship of daily hot water bathing at home and hot water spa bathing with underlying diseases in middle-aged and elderly ambulatory patients: A Japanese multicenter cross-sectional study. Complement Ther Med 2019;43:232-9.

(c) 2019 Association for Research into Arterial Structure and Physiology. Publishing services by Atlantis Press International B.V. This is an open access article distributed under the CC BY-NC 4.0 license (http://creativecommons.org/licenses/by-nc/4.0/). 